\title{
Sme//Control: The Study of Sense of Agency in Smell
}

\author{
Patricia Cornelio $^{1}$, Emanuela Maggioni ${ }^{1}$, Giada Brianza ${ }^{2}$, Sriram Subramanian ${ }^{1-2}$, Marianna Obrist ${ }^{1-2}$ \\ ${ }^{1}$ University College London, ${ }^{2}$ University of Sussex \\ United Kingdom \\ \{p.cornelio, e.maggioni, s.subramanian,m.obrist\}@ucl.ac.uk, g.brianza@sussex.ac.uk
}

\begin{abstract}
The Sense of Agency (SoA) is crucial in interaction with technology, it refers to the feeling of 'I did that' as opposed to 'the system did that' supporting a feeling of being in control. Research in human-computer interaction has recently studied agency in visual, auditory and haptic interfaces, however the role of smell on agency remains unknown. Our sense of smell is quite powerful to elicit emotions, memories and awareness of the environment, which has been exploited to enhance user experiences (e.g., in VR and driving scenarios). In light of increased interest in designing multimodal interfaces including smell and its close link with emotions, we investigated, for the first time, the effect of smellinduced emotions on the SoA. We conducted a study using the Intentional Binding (IB) paradigm used to measure SoA while participants were exposed to three scents with different valence (pleasant, unpleasant, neutral). Our results show that participants' SoA increased with a pleasant scent compared to neutral and unpleasant scents. We discuss how our results can inform the design of multimodal and future olfactory interfaces.
\end{abstract}

\section{CCS CONCEPTS}

- Human-centered computing $\rightarrow$ Human computer interaction $(\mathrm{HCI}) \rightarrow$ Empirical studies in $\mathrm{HCI}$.

\section{KEYWORDS}

Smell; Olfaction; The Sense of Agency; Intentional Binding; Olfactory Interfaces; Emotions; Multimodal; Multisensory.

\section{ACM Reference format:}

Patricia Cornelio, Emanuela Maggioni, Giada Brianza, Sriram Subramanian and Marianna Obrist. 2020. SmellControl: The Study of Sense of Agency in Smell. In 2020 International Conference on Multimodal Interaction (ICMI'20), October 25-29, 2020, Virtual Event, Netherlands. ACM, New York, NY, USA, 11 pages. https://doi.org/10.1145/3382507.3418810

\section{INTRODUCTION}

Our sense of smell has a proven effect on our emotions and is often referred to as emotional system [26]. Imagine how it makes you feel to smell the warm, sweet scent of hot chocolate on a cold

Permission to make digital or hard copies of all or part of this work for personal or classroom use is granted without fee provided that copies are not made or distributed for profit or commercial advantage and that copies bear this notice and the full citation on the first page. Copyrights for components of this work owned by others than ACM must be honored. Abstracting with credit is permitted. To copy otherwise, or republish, to post on servers or to redistribute to lists, requires prior specific permission and/or a fee. Request permissions from Permissions@acm.org.

ICMI'20, October 25-29, 2020, Virtual Event, Netherlands

(c) 2020 Association for Computing Machinery.

ACM ISBN 978-1-4503-7581-8/20/10...\$15.00

https://doi.org/10.1145/3382507.3418810 winter morning versus an unpleasant body odor of a stranger sitting next to you on the train. Smell is also powerful to trigger memories, especially for childhood memories [47]. In light of the power of smell for influencing our experiences, multimodal interfaces are increasingly integrating olfactory signals as a novel interaction modality for creating more emotionally engaging experiences [75].

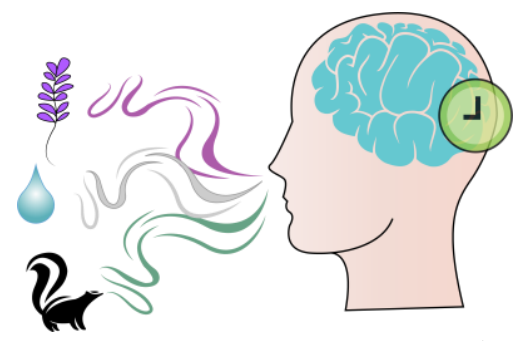

Figure 1: Studying the effect of pleasant (lavender), unpleasant (civet), and neutral (water) scents on the SoA.

While our understanding of how smell can be used in our interaction with technology is rapidly increasing (e.g., smell can be used to convey messages [30,62], induce alertness [93] and increase awareness of the environment by facilitating location detection [44] and enhancing realism [78]), it is still unknown how smell affects our Sense of Agency (SoA) i.e., the feeling of being in control in the environment [41].

The SoA has been suggested to be crucial in our interaction with technology [60]. A recent study has shown that if a system does not support agency, the user might feel discouraged from using it [61]. Within human-computer interaction (HCI) research, it has been shown that on-skin input [45] produces higher SoA than keyboard and touchpad inputs [11,24], and that auditory and haptic feedback elicit higher SoA than visual feedback only [21]. However, the role of smell on agency remains unknown.

Apart from a growing body of knowledge demonstrating how agency is modulated by different interaction modalities (visual, auditory, haptic), there is also growing evidence that agency is subject to affective modulation [95]. That is, people tend to feel higher SoA when a positive emotion is involved rather than a negative emotion [1, 13, 20, 88, 95]. However, these studies have modulated people's emotions using visual [1], auditory [20, 95] and tactile [12] cues, and the role of olfactory cues (emotions induced by smell) on agency has not been explored.

In this paper, we bridge this gap in our understanding of SoA and smell and investigate the specific effect of smell-induced emotions on the SoA. We conducted a user study to explore this effect using the established Intentional Binding (IB) paradigm that provides an implicit metric of the SoA [42]. We primed participants with three different emotions (positive, negative, and neutral). Positive and negative emotions were modulated by the 
exposure to a pleasant (lavender) and an unpleasant (civet) scent respectively (see Figure 1). A neutral emotion was induced using a baseline scent (water). Our results show an increased SoA when participants were exposed to the pleasant scent compared to the unpleasant and neutral scents. These findings support that smell produces not only physiological responses [90] and modifies emotions $[86,91]$ but also show for the first time that smell affects the feeling of agency.

Smell is an emerging and unexplored sense in multimodal interfaces. Our motivation lies in recent efforts to exploit smell as interaction modality. For example using scents as output for HCI (olfactory displays [51, 52]) and as a system outcome (olfactory notifications [30,62]). Shifting users' SoA through smell can be useful in these emerging olfactory interfaces, where scents represent a response from the system that makes user feel in control. Our study delivers for the first-time evidence of the effects of smell on agency and thus enriches prior work on agency and visual, auditory and haptics interfaces.

With this paper we make a threehold contribution: (1) we explore the relationship beween agency and the sense of smell, an increasingly relevant interaction modality within multimodal interaction design, (2) we investigate the specific link between smell and emotions on SoA based on the growing literatue of affective modulation, and (3) discuss the relevance of our results for the design of future smell-based applications.

\section{LITERATURE REVIEW}

\subsection{The Sense of Agency}

The Sense of Agency (SoA) refers to the experience of being the initiator of one's own voluntary actions (being an agent) and through them influence the world around us [12]. The SoA has been suggested to reflect an experience of being in control. For example, when we switch the light on and we perceive the light coming on (i.e., I did that!) or when we press a key from the keyboard and the computer responds with a visual effect onscreen (i.e., I control this!). The SoA is gaining increasing attention from the field of HCI aiming to advance our understanding of the role of agency in the use of technology. Developing user interfaces that increase the user's SoA will provide users with the feeling of "I did that" as opposed to "the system did that", supporting thus an internal locus of control (a key rule for user interfaces [84]).

The SoA is commonly measured using explicit questionnaires, by simply asking the user whether she was the agent of certain action or not (e.g., "did you do that?") [43], or subjective scales (e.g., rate your level of control on a scale from 1 to 7 ). However it can be implicitly measured using the Intentional Binding (IB) paradigm [42], which links agency experience and perception of time. This paradigm indicates that the interval between a voluntary action and its outcome is perceived as shorter than it actually is. This is illustrated in Figure 2(a) right, when subjects perform a voluntary action (e.g., press a button) and this action causes an outcome (e.g., a tone), they tend to perceive delayed awareness of the action (called action binding) whilst anticipated awareness of its outcome (called outcome binding). In other words, people tend to perceive voluntary actions and their outcomes shifted towards each other [34, 42]. The summation of action binding and outcome binding (called total binding) is thus associated to the experience of agency. The higher the total binding, the higher the SoA $[34,68]$.

To measure subjects' time perception, a Libet clock on-screen (Figure 2(a) left) is used, it consists of a clock that rotates clockwise once every $2560 \mathrm{~ms}$ [58, 59]. Subjects are asked to look at the clock's rotating dot and report the time (the dot location) at the moment when they performed the action or the moment when they perceived the outcome.

As shown in Figure 2(b), four different conditions are employed to calculate action binding and outcome binding (two baseline and two active conditions). In the baseline conditions, only one event occurs either action or outcome. In the active conditions, both action and outcome occur.

During the task, both actual time (i.e., the dot position on the Libet clock logged by the system) and perceived time (the dot position on the Libet clock reported by subjects) of the action and outcome are recorded. The errors are then calculated by the difference between perceived and actual time in milliseconds. Following this, the Intentional Binding (total binding) is calculated through the formulas shown in Figure 2(c).

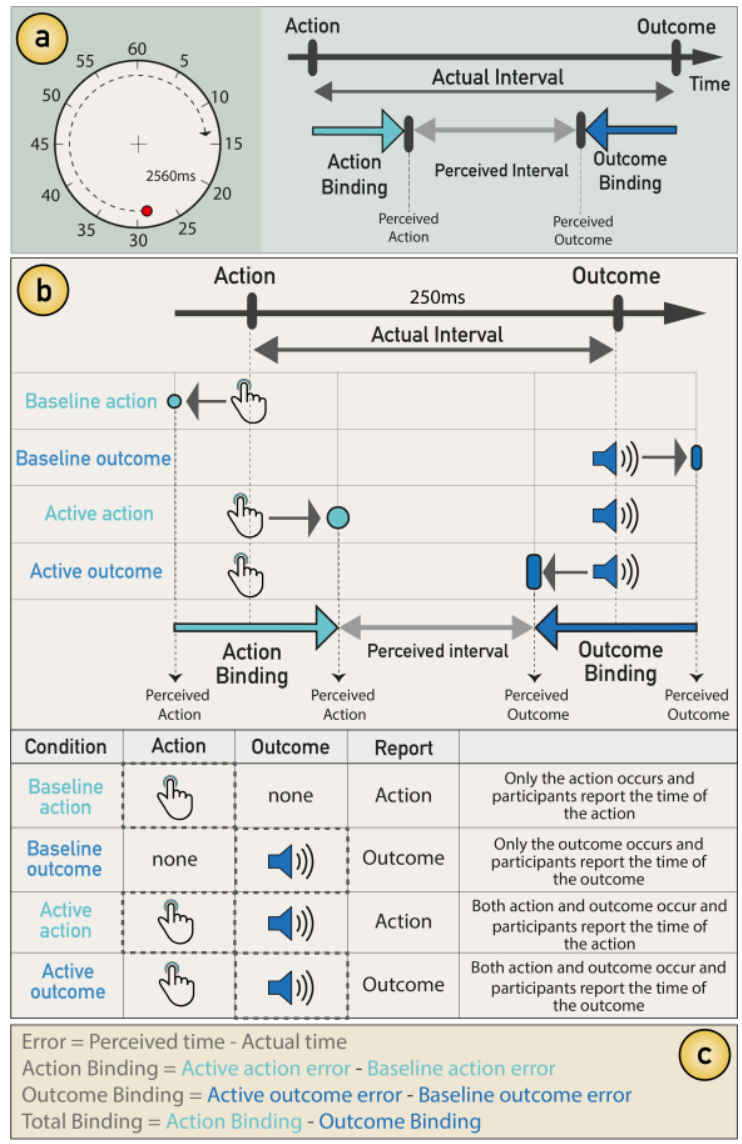

Figure 2. (a) Left: The Libet clock. (a) Right: The Intentional Binding (IB) effect. (b) Measurement blocks (baseline \& active). (c) IB calculation formulas. 
In summary, this paradigm measures the extent to which a voluntary action and its effect subjectively shift together across time, to determine the source of action (I did it). This can be depicted as the bi-directional limitation of Bayesian causal inference [33]: "if two events occur closer together in time, it is more likely they will be perceived as causally related. Therefore, if two events are known to be causally related, they are more likely to occur closer in time" [17].

Since the IB paradigm has been widely employed in the literature; demonstrated to be a viable metric to implicitly assess the SoA [67], and it has been recently employed in HCI to measure agency for different input commands and system feedback [11, 21, 24], we employed this method for our study.

\subsection{Smell as Interaction Modality}

Although our everyday activities involve five basic senses, our interaction with technology is dominated by visual, auditory and haptic I/O channels. However, recent research that pursues multisensory experiences in $\mathrm{HCI}$ [73] has revealed relevant opportunities for smell [75] in user interfaces.

For instance, olfactory stimulation has gained attention in driving scenarios [93]. Dmitrenko et al. [30] explored odors as information medium by mapping scents onto messages from the car. For example, the "slow down" message was strongly associated to lemon odor while "fill gas" to peppermint odor. These olfactory messages allow drivers to receive information from the car while other senses are engaged with additional information (e.g., listen to the radio and focus on the road).

Yoshida et al. [93] explored olfactory stimuli to alert drivers in drowsy driving and prevent fatal car accidents. They found that peppermint fragrance effectively induces wakefulness. In another example Baron et al. [9] found that lemon scent improves drivers' alertness. Smell notification systems have been even patented by car companies such as Ford [54].

In the line of desktop messaging, Maggioni et al. [62] explored olfactory notifications that serve as feedback from the system. They associated scents with different message senders alleviating visual attention and found that olfactory notifications improve users' performance in identifying the urgency level of a message.

Odors also enhance the sense of presence [7] and realism [66] in Virtual Reality (VR). Scents presentation leads to an increased sense of reality and relevance [38]. For instance, a study showed that tactile, olfactory and auditory cues produce more sense of presence and memory than just adding visual details in the scene [27]. In other example, Narumi et al. [71] employed different scents to create the illusion of flavors in augmented reality.

Olfactory interfaces are covering many others application scenarios such as, gaming [66, 70], rehabilitation [23] multimedia $[15,64]$ and communication [79, 98]. However, despite this increasing inclusion of smell in $\mathrm{HCI}$ and the increasingly considered importance of the SoA in interaction with technology, previous research studying agency in HCI has mainly focused on visual, auditory and haptic interfaces, therefore, the role of smell on the SoA has remained unexplored. We address this limitation by studying the relationship between smell and agency through their close link with emotions. In the next section, we explain this link by reviewing literature that has shown the relevance of emotions on agency (affective modulation) and smell.

\subsection{Relevance of Emotion on Agency and Smell}

Here we describe the known relationship between agency and emotions and between emotions and smell.

\subsubsection{Effect of Emotions on Agency}

The self-attribution process of the SoA (e.g., I did that) is influenced by affective information [5, 6, 13, 40]. People tend to attribute positive events to their own actions (self-attribution), while negative events are usually attributed to external agents. In other words, take the credit for positive events and blame external factors for negative events (the so called self-serving bias [4, 65]).

For instance, Takahata et al. [88] primed subjects with rewarding and punishing action's outcomes by associating auditory stimuli with positive, neutral and negative monetary outcomes. They found that participants exhibited higher SoA for positive outcomes (monetary gains) unlike negative outcomes (monetary losses). Yoshie and Haggard [95] manipulated the emotional valence of action outcomes with negative or positive emotional vocalizations [81] or neutral tones. They found that SoA was reduced for negative compared to positive or neutral outcomes. Christensen et al. [20] investigated the influence of action outcome valence (modulated by emotional human vocalisations) on prospective and retrospective components of agency. They found that positive outcomes enhanced the retrospective agency for unexpected outcomes. Borhani et al. [12] found that having control over negative somatosensory outcomes (induced by painful heat or electrical stimulus) increases the SoA.

In another example, Aarts et al. [1] explored affective priming through visualization of emotional pictures [57]. Unlike the above examples, here the emotional information was not presented as an action outcome, but in form of priming i.e., it consisted of a brief exposure to pictures (positive and neutral) before an IB task. They found that positive reward signals via positive pictures enhance the SoA unlike neutral pictures.

These studies reveal modulation of agency depending of emotions. However, they have modulated the valence of information (positive, negative or neutral) using visual, auditory and tactile information, therefore modulation of agency via smellinduced emotions remains unknown despite the power of smell to modulate affective states.

\subsubsection{Effect of Smell on Emotions}

There is a close link between smell and affective information. Odors not only evoke strong experiences of pleasure or displeasure [35], but also modulate mood [91], attention [53, 89], stress [3, 69] and memories [47]. Different scents have been shown to elicit specific physiological responses or emotional states. For example, lemon is linked with arousal [31] while lavender is considered a relaxing scent [69]. Lemon odor has also been suggested to have antidepressant properties [55] while lavender and rosemary decrease cortisol levels in saliva [3]. Lavender is associated with happiness while acetic acids are linked to anger and disgust [90]. 
The olfactory system is deeply linked to areas of the brain that regulate emotions [86, 96]. For that reason, emotions evoked by odorants are very strong. For instance, it has been suggested that "odor-evoked memories are more emotional than memories evoked by other sensory stimuli" [47] and also that people who have lost their sense of smell depress more than people who have lost their vision [85]. While the sense of smell is often considered as a poor sense [83], emerging research interestingly suggests that we use it more than we actually think. For example, a study revealed that humans have scent tracking abilities similar to dogs [77] and also that emotions can be communicated via olfactory channel so that we can smell someone's fear [25].

Furthermore, it has been recently shown that smell produces an effect on Body Image Perception (BIP). BIP refers to "perceptions, attitudes and beliefs pertaining to one's own body" [49], comprising emotional attitudes towards it. For instance, Brianza et al., [16] found that lemon scent is significantly associated with a thin body while vanilla with a thick body. They also showed subjective reports of feeling lighter when smelling lemon and heavier when smelling vanilla. Their results further revealed that, combining lemon scent and high pitch footsteps sound produced subjective reports of walking faster, feeling lighter and having a straighter posture unlike vanilla scent. This suggests that positively influencing BIP with smell can be reflected in feelings of being more positive [87].

Those findings show a strong potential of smell for helping not only in user interfaces but also in people's wellbeing.

\section{USER STUDY: IS AGENCY INFLUENCED BY SMELL?}

The emotional content of events (modulated by audio, vision and touch) can influence the experience of agency. These findings open up an opportunity to explore agency modulation through other senses. Given that agency is crucial for the interaction with technology and can be modulated by emotions, we wanted to explore if agency is affected by a powerful sense that is strongly connected to our emotions: the sense of smell.

To explore this, we conducted a user study that explored the effect of positive, negative and neutral olfactory cues on the SoA. It has been shown that scents can influence performance, attention, pleasure, alertness or message urgency (as shown in the literature review), therefore, in this study we aim to investigate if the feeling of control can be influenced by odors. Particularly, following the literature, we hypothesize that a pleasant odor will increase the SoA in comparison with an unpleasant odor.

During the study, we primed participants with three different types of emotion (positive, negative and neutral) at the beginning of an IB task as in Aarts work [1]. However, here we presented participants with three scents: lavender (pleasant), civet (unpleasant) and water (neutral). We measured three main variables in our study. First, in order to evaluate whether our scents produced the intended emotions on participants, we assessed explicit emotion using a Self-Assessment Manikin (SAM) scale [14], commonly employed to study the three dimensions of emotions (valence, arousal and dominance) during smell stimulation [80]. Second, since previous studies have suggested that smell stimulation produces an activation of the neural central system reflected in changes on physiological signals such as Galvanic Skin Response (GSR) [90], we also measured skin resistance in order to evaluate whether our scents produced such activation. Third, we assessed implicit agency by measuring IB as an indicator of the SoA modulation [42]. In the next sections we describe the methodology for emotion elicitation and IB task.

\subsection{Emotions Induced by Smell}

The fragrances we used are $100 \%$ pure essential oils not diluted. For positive emotion, we chose lavender scent (available in Plush Folly [76]) as it has been widely employed to produce pleasant emotions [90, 91]. For negative emotion, we chose civet scent (available in Holland \& Barret [10]) which is often used for perfumes base, and in its pure state, is considered unpleasant since it is the perineal gland secretion produced by the civet cat $[50,82]$.

We are aware of cultural variability in odor perception. For that reason, we particularly chose lavender and civet scents since they have been effectively used for inducing positive and negative emotions [80,97] and demonstrated to be effectively categorized as pleasant and unpleasant between cultures: French, Vietnamese \& American [19] and Switzerland, UK, \& Singapore [37] showing no big differences between gender.

Finally, we employed water to induce a neutral emotion following previous work using it as a control stimulus $[8,28]$.

\subsubsection{Scent Delivery}

Figure 3 shows the structure of the scent delivery system. It consisted of a custom-made electronic device controlled by an Arduino board. The device is composed of 3 electro-valves $(4 \mathrm{~mm}$ Solenoid/Spring pneumatic valve) that regulate the air passage (on-off) from a tank of compressed air. The tank (701/s, maximum pressure of 8 Bar) supplied air flow through $4 \mathrm{~mm}$ plastic pipes passing by the electro-valves and arriving to three small glass bottles that contained the two essential oils (lavender and civet) and water (our baseline scent) respectively. The tank airflow was set at a constant pressure of $1 \mathrm{Bar}-1 / \mathrm{min}$, through an air regulator. This device was built following the guidelines from [29].

The odours reached the participant through a 3D-printed nozzle $(\varnothing=3.5 \mathrm{~cm})$, that was positioned at $30 \mathrm{~cm}$ from the participants' nose [29], and never directly in contact with participants to avoid an air puff sensation. With our setup (see Figure 4), the scents take about 1.5 seconds to be perceived (from the valve triggers to participants judgement) as revealed in a pilot study we conducted before the actual experiment. During all the experiment participants rested their chin on a supporting structure critically positioned to ensure scent delivery towards participants' nose (i.e., they adopted the same position during all the conditions).

\subsubsection{Subjective Emotional Evaluation}

After each scent condition, a SAM scale [14] was used to measure the three dimensions of emotion. i.e., valence, arousal and dominance reported by participants for each scent. It consisted of a 9-points scale for each dimension. A value of 9 represents a high score while a value of 1 a low score in each dimension. 


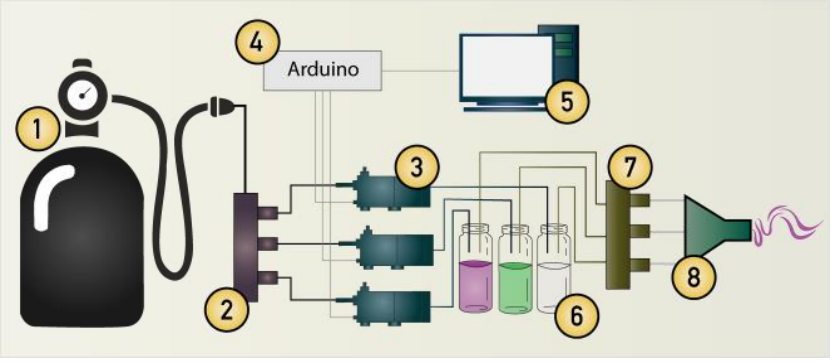

Figure 3. Structure of the scent delivery system: (1) air tank, (2) manifolds, (3) electro-valves, (4) Arduino, (5) PC,

(6) glass bottles with the scents, (7) one-way valves, (8) output nozzle.

\subsubsection{Physiological Activation (skin resistance)}

Skin resistance was measured using a Shimmer3 GSR+ Unit wireless device (Shimmer Sensing, Dublin). Participant wore an armband with the shimmer device attached and two $8 \mathrm{~mm}$ snap style finger electrodes on their index and middle fingertips (with a constant voltage $0.5 \mathrm{~V})$. We recorded data with a frequency of $512 \mathrm{~Hz}(10 \mathrm{mSiemens}(\mu \mathrm{S}) /$ Volt, A/D resolution of $12 \mathrm{bit})$ allowing to record responses ranging from 2 to $100 \mu \mathrm{S}$. We recorded skin resistance during each scent condition (neutral, pleasant and unpleasant) for 5 seconds in each trial. Additionally, we measured skin resistance during a baseline condition consisting of 60 seconds prior to the experiment without the exposure to any scent to compare baseline recordings with our experimental conditions.

The synchronization between smell delivery and skin resistance measurement was computer-controlled following the sequence shown in Figure 5. Our interface was programmed to release the scent upon participants pressing a footswitch which we also used as a marker to start recording GSR in each trial.

Before statistical analysis, we pre-processed the data for downsampling and movement artefact identification and correction after careful inspection. Our GSR measurement refers to a general signal containing both components the tonic and phasic.

\subsection{Intentional Binding (IB) Task Procedure}

To measure SoA, we employed the traditional desktop IB task consisting of a button-press voluntary action and a tone as its outcome. Every trial started when participants pressed a footswitch and a fixation cross appeared on-screen while the scent was being presented during 2500ms. After participants were primed with the scent cue, a Libet clock was shown and always started rotating from a random position. Then, participants were asked to press a button (space bar from a keyboard) at elapsed time of their preference (voluntary action). After a fixed interval of $250 \mathrm{~ms}$ they heard a tone (the action's outcome) which lasted $100 \mathrm{~ms}$ at $900 \mathrm{~Hz}$. Subsequently, after a random interval between $1000 \mathrm{~ms}$ and $1500 \mathrm{~ms}$ the clock stopped and participants were asked to report the position of the clock (where the dot was) at the moment when they either executed the action (baseline action and active action blocks) or perceived the tone (baseline outcome and active outcome blocks) as described in Figure 2(b). Figure 5 shows the procedure of a single trial.

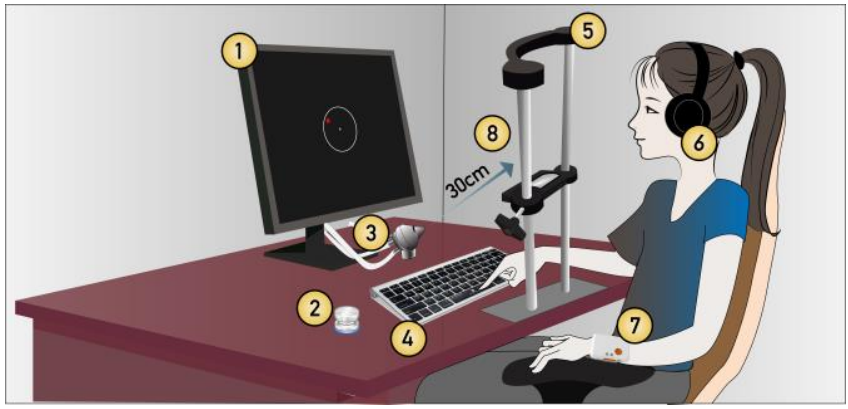

Figure 4. Task setup: (1) Libet clock on-screen, (2) USB controller, (3) output nozzle, (4) keyboard, (5) chinrest, (6) headphones, (7) GSR sensor, (8) $\mathrm{D}=30 \mathrm{~cm}$.

The Libet clock rotated clockwise once every $2560 \mathrm{~ms}$, its size was 500 pixels, shown on a screen of 24-inch, 1920 x 1200 in resolution. The numbers of the clock were removed in order to avoid creating visual patterns during the task [21, 22]. Instead, after each trial when the dot stopped, participants used a rotational controller (Griffin Powermate Knob) to relocate the dot on their perceived position. We then calculated time errors by the difference between perceived and actual clock positions and calculated IB (action binding, outcome binding and total binding) using the formulas shown in Figure 2(c).

Participants performed 4 blocks (described in Figure 2 b) of 20 trials each in each scent condition (3 types), resulting in 240 trials per participant. Between scent conditions, we used an air extractor in the room for about $3 \mathrm{~min}$ to clear the environment. We limited the number of trials to 20 to avoid making the experiment too long, and to follow the number of trials used in previous IB studies [21]. The full experiment took about $90 \mathrm{~min}$ in total including $3 \mathrm{~min}$ break between conditions (air cleaning). Each scent condition (consisting of 20 trials and 4 blocks), took about $20 \mathrm{~min}$. We considered that a longer duration could have produce a habituation effect. Participants wore noise-cancelling headphones playing white noise during all the experiment to block sounds from the devices.

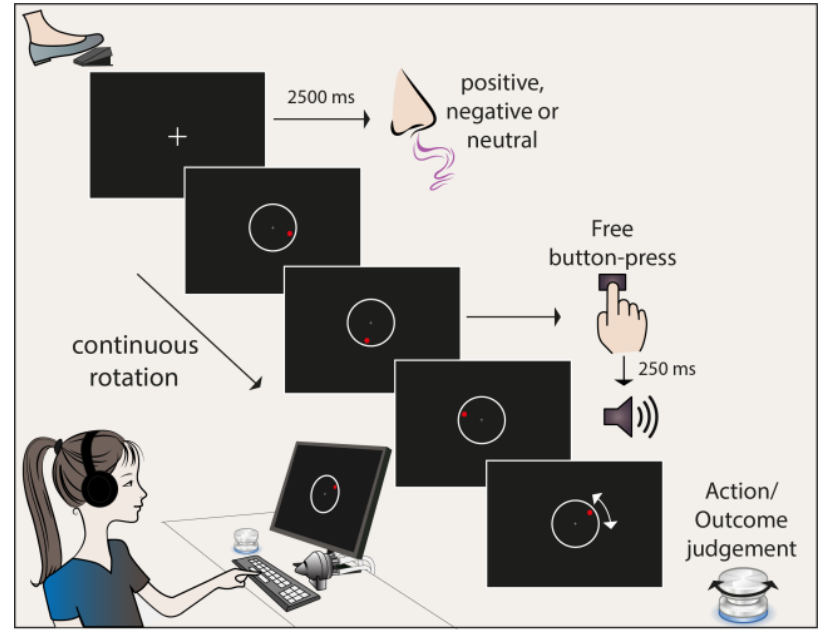

Figure 5. Procedure of the IB task. 


\subsubsection{Participants}

Thirteen right-handed participants $(4$ females, mean age $=31.39$ years old, $\mathrm{SD}=5.33$ ) took part in our study. They had normal or corrected-to-normal vision and were prescreened prior to the experiment using an olfactory assessment test [72] (see supplementary material) to make sure their sense of smell was not impaired and that they were not suffering from allergies, cold or flu. Females during their menstrual cycle or pregnancy were excluded as hormone levels change olfactory sensitivity [18, 32]. The local ethics committee approved this study.

An a priori statistical power analysis was performed for sample size estimation in $G^{*}$ Power. Running a power analysis on a repeated measures ANOVA with three scents conditions (neutral, pleasant, and unpleasant), repeated 4 times (4 blocks of the IB paradigm), a power of 0.95 , an alpha level of 0.05 , and a medium effect size $\left(\mathrm{f}=0.25, \eta_{\mathrm{p}}{ }^{2}=0.06\right.$, critical $\left.\mathrm{F}=1.63\right)[36,56]$, requires a sample size of about 12 participants. Thus, our proposed sample of 13 participants was adequate for the objective of this study.

\subsection{Results}

\subsubsection{Results on Emotion Elicitation}

A One-way Repeated Measures ANOVA for each dimension of emotions (valence, arousal and dominance) was conducted to compare the effect of each scent (pleasant, unpleasant and neutral) on participants scores from the SAM scale. Results showed significant effect of scents on valence $\left(\mathrm{F}_{(1,11)}=4.65, p<0.05\right.$, $\eta_{\mathrm{p}}{ }^{2}=0.28$ ), comparison tests with Bonferroni correction showed a significant difference between all the three scents (Figure 6 shows mean scores). A significant effect on arousal was also shown $\left(\mathrm{F}_{(1,11)}=4.87, p<0.05, \eta_{\mathrm{p}}{ }^{2}=0.22\right)$, comparison tests with Bonferroni correction showed a significant difference between neutral and pleasant $(p<0.05)$ scents and between neutral and unpleasant $(p<0.05)$ scents. A non-significant effect of scents on dominance is shown $\left(\mathrm{F}_{(1,11)}=0.144, p=0.71, \eta_{\mathrm{p}}{ }^{2}=0.01\right)$.

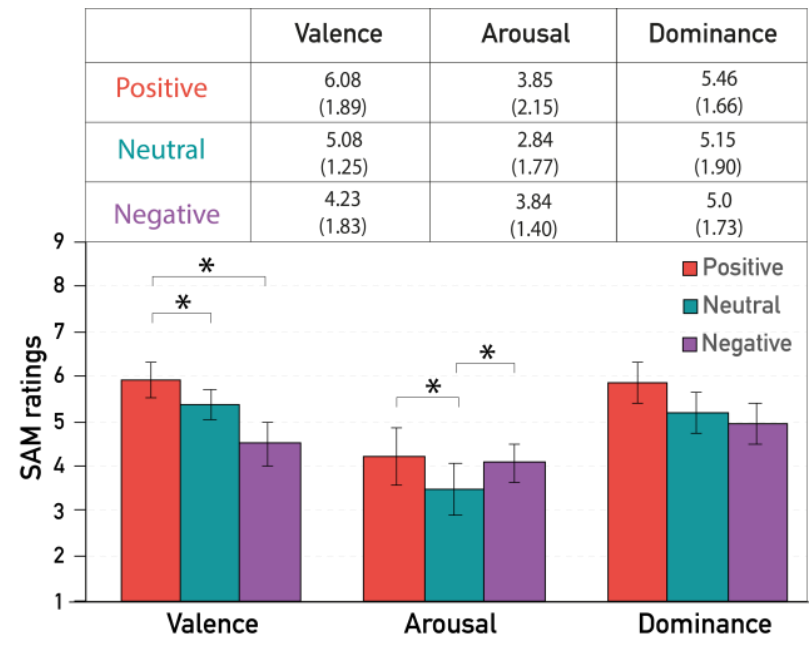

Figure 6. Mean scores of valence, arousal and dominance using the SAM scale (rated with a 9-points scale) grouped by scent type with \pm SD in brackets. Error bars represent SEM. ${ }^{*}=\mathbf{p}<0.05$ in valence.
These results show that the scents employed in our study, effectively produced the intended emotions on participants (positive, negative and neutral). Participants scored the lavender scent significantly higher in valence compared with the civet and water scents, while the civet scent was reported with the lowest valence scores. In terms of arousal, the lavender scent was overall scored with low arousal (see Figure 6) which supports that lavender odor is considered relaxing [69]. However, water, used as neutral stimulus, was reported significantly less arousing than the lavender and civet scents.

\subsubsection{Results on Physiological Activation}

One-way Repeated Measure ANOVA was conducted to compare the effect of the scent stimulation on skin resistance reactions. Results showed significant effect of scents on skin resistance $\left(\mathrm{F}(1,11)=4.34, p<0.05, \eta_{\mathrm{p}}^{2}=0.28\right)$. Particularly, comparison tests with Bonferroni correction showed a significant difference between the baseline and all the scents conditions $(p<0.01)$. In line with prior work, this suggests that participants' skin resistance was affected by the elicited emotion which confirms that there was a response from the neural central system due to the scent stimulation [ 48 , 92]. However, no significant effect is shown between the three emotional scents (pleasant, unpleasant and neutral). Figure 7 shows the mean skin resistance in Ohms.

While we expected to see differences in GSR between scents, we mainly focused on the comparison between baseline (no scent) and the scents conditions. We interpret GSR changes as an effect of momentary activation in response of a temporary external trigger. The stimulation occurred in such a short time that the valence connotation of the scents could have been not detected; this probably would have been shown in the tonic component of the GSR if the scent stimulation would have been longer.

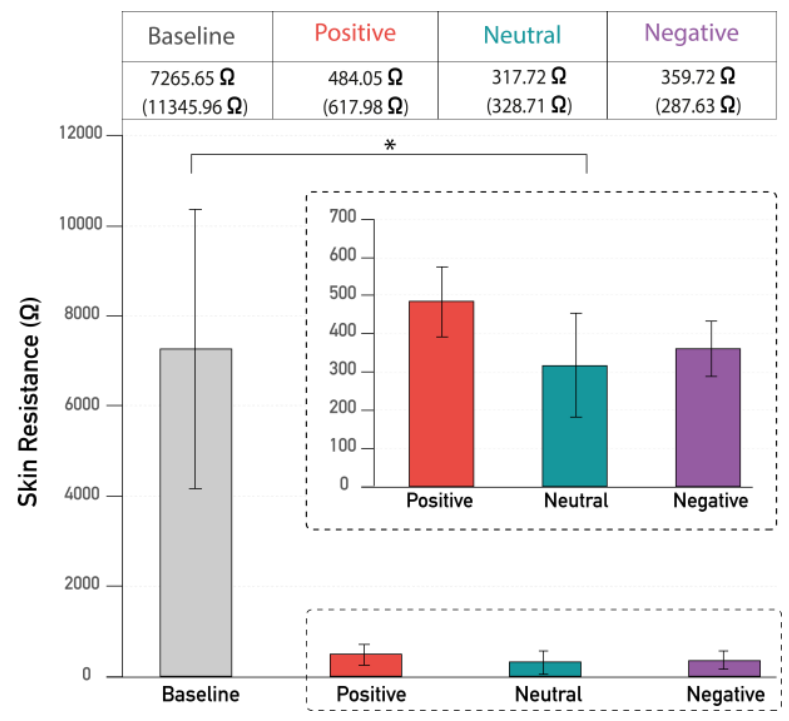

Figure 7. Mean skin resistance in Ohms $(\Omega)$ with \pm SD in brackets grouped by scent type and the baseline condition (no scent exposure). The upper dashed rectangle shows a zoom of the emotional scents. ${ }^{*}=\mathbf{p}<0.05$. 


\subsubsection{Results on SoA (Intentional Binding)}

A One-way Repeated Measures ANOVA was conducted to compare the effect the scents used as emotional prime (i.e., pleasant, unpleasant and neutral) on action, outcome, and total binding. Results showed significant effect of the emotional prime on the total binding $\left(\mathrm{F}_{(1,11)}=4.50, p<0.05, \eta_{\mathrm{p}}{ }^{2}=0.26\right)$. Particularly, comparison tests with Bonferroni correction showed a significant difference between the neutral and pleasant scent $(p<0.01)$ and between the pleasant and unpleasant $(p<0.05)$ scents. Results also showed a significant effect of the emotional scent on the outcome binding $\left(\mathrm{F}(1,11)=5.53, p<0.05, \eta_{\mathrm{p}}^{2}=0.30\right)$, comparison tests with Bonferroni correction showed a significant difference between pleasant and neutral $(p<0.01)$ and the pleasant and unpleasant scents conditions $(p<0.05)$. However, for action binding $\left(\mathrm{F}_{(1,11)}=1.08, \quad p=0.05, \eta_{\mathrm{p}}^{2}=0.16\right)$ we found no significant effect between scents. Details related to mean time in relation to action, outcome, and total binding in each of the emotional prime condition are presented in Figure 8.

\subsubsection{Effect of Emotions Induced by Smell on the SoA}

Based on the study results we can see that participants exhibited significantly higher total IB in the pleasant scent condition. This suggests that participants felt higher SoA during the task when they were primed with the lavender scent compared with the civet and water scents. These findings are in accordance with prior research on modulation of agency through emotional cues i.e., people tend to self-attribute events when positive information is involved unlike negative information (the self-serving bias [4]). Although previous studies have demonstrated this by modulating the valence of emotions (positive, negative or neutral) using visual (e.g., pictures [1]), auditory (e.g., human vocalizations [20, 94, 95]) and tactile (e.g., cutaneous heat-pain [12]) cues, in the present study we show that the experience of agency is modulated by olfactory cues, being higher when a pleasant scent is presented.

As shown in Figure 8, we found no differences in action binding (the delayed awareness of the action) among the different emotional conditions. However, we observed significantly higher outcome binding (the anticipated awareness of the outcome) with the pleasant scent in contrast with the neutral and unpleasant scents. This is in line with the work from Aarts and Haggard [1, 12], where the main effect was observed on the outcome binding. This suggests that the positive signal via a pleasant scent caused a stronger prediction of the outcome. Yet, further studies are needed to explore pleasant scents with high arousal on the SoA, since lavender is considered a relaxing scent.

We observed a low IB in the neutral condition. A possible reason of this low value might be related to the challenges of presenting a "neutral scent". Even when we used water instead of essential oils, and the elements in our delivery system (e.g., vales, manifolds, bottles) were completely independent in each scent condition (see Figure 3), participants might have smelled components from the device. Indeed, two participants reported have perceived the neutral scent as a slightly plastic-like scent which could be caused by the plastic tubes transporting the air or the 3D-printed nozzle. Although we expected to observe higher IB in the water condition, the main effect from the pleasant scent is clearly observed in comparison with the other two conditions (see Figure 8). However, we recommend using odorless materials for delivery of neutral scents (e.g. glass tubes) to avoid odors perceived from the devices.

\section{DISCUSSION AND OPPORTUNITIES FOR MULTIMODAL INTERFACES}

A feeling of being in control is crucial in designing interactive systems and multimodal interfaces [84]. That is, designing systems that give users the feeling of "I did that" rather than "the system did that" gives a more agentive and responsive experience. In this paper, we have extended the current state-of-the-art knowledge on the study of agency and agency modulation. While previous studies have studied agency modulation through novel input modalities (e.g., speech [61] and gestural [21]), and system feedback (e.g., mid-air haptics [21]) in the present paper, we have introduced smell as interaction modality into the study of agency.

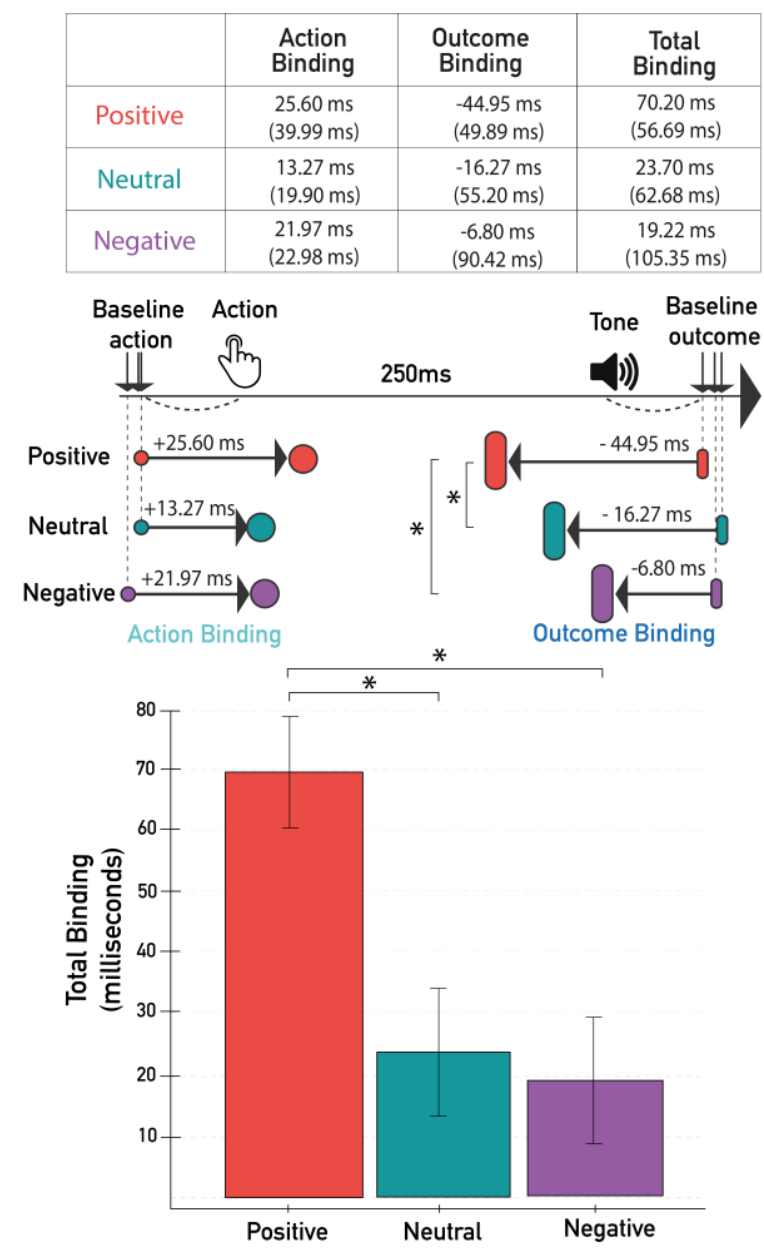

Figure 8. (Top) Mean action, outcome and total binding in milliseconds with \pm SD in brackets. (Middle) A positive value represents a delayed awareness (action binding) while a negative value an early awareness (outcome binding). (Bottom) Total binding (Action Binding Outcome Binding). Error bars represent SEM. ${ }^{*}=\mathbf{p}<0.05$. 
Unlike other sensory modalities (e.g., vision, audio or touch) the sense of smell provides a direct path between olfactory cues and the amygdala [96]. Clinical research indicates that "emotional behavior is critically dependent on the amygdala" [2]. Our results provide evidence of agency modulation via a channel that is directly connected to our emotions, suggesting thus that an increase on the SoA is unlikely to be produced by additional parameters that accompany other sensory modalities. However, since our study focused on smell only, further work is needed to demonstrate this by comparing different sensory modalities.

Our results thus open up opportunities for designing user interfaces that improve user's SoA through multisensory signals. Although the sense of smell is often considered poor [74, 83] and its inclusion in HCI seems challenging [39] (especially in light of scent delivery [29]), in this study we have demonstrated that the feeling of controlling the environment increases when people are exposed to a pleasant scent.

For example, we could use our results to discuss and explain why fragrance presentation is known to improve performance in driving scenarios [9]. Presenting a pleasant scent such as lavender, could help drivers to feel more in control about the in-car interaction involving voluntary actions and responses from the car. Preserving a SoA in driving scenarios is also important in the context of autonomous vehicles, where agency is shared between the driver and the system. Hence olfactory stimulation could be an option to stablish an environment to not only induce alertness [93], improve mood [46] and help angry drivers [28], but also help enhancing the feeling of being in control.

In another example, we can imagine that smell stimuli in VR environments cannot only improve realism, immersion [78] and awareness of the environment [44], but also provide the user with a stronger feeling of control in this digital space. VR further affords the opportunity to not just modulate but potentially manipulate SoA through changing the scent and also other sensory features in a virtual world, and thus create completely new experiences.

There are many more application examples imaginable, we hope our results will inspire. We believe that advancing the understanding of how smell can be used in our interaction with technology, can increase the inclusion of olfaction as a novel interaction modality and facilitate the design of future olfactory interfaces by giving designers a different lens to design for.

In addition, since the SoA has been suggested to underpin the concept of responsibility in human societies [43], our paper also highlights the relevance of agency measures in user interfaces evaluation. That is, our sense of being an agent is not only related to executing motor actions but also to knowing "the nature and quality of the act" [41]. Therefore, it is important that systems give users the appropriate feeling of control in order to preserve a feeling of responsibility (especially in critical situations such as driving). In other words, it is important that systems let users clearly experience what they are doing and therefore self-attribute events. We believe that agency measures should be included within user experience (UX) metrics to design systems that not only consider usability but that also support both a feeling of being an agent and a feeling of being responsible for events.
Overall, our paper contributes new knowledge to the study of SoA and the use of smell as interaction modality. As we increasingly design for smell in multimodal and multisensory interfaces (for work, education, relaxation, health or wellbeing [63]), it is important that designers and developers not only in academia but also in industry are more aware of important underlying concepts such as the SoA. Being the agent of an action is increasingly important in a time where intelligent algorithms make decisions for us and influence knowingly or unknowingly our behavior in an increasingly digital world.

\section{LIMITATIONS AND FUTURE WORK}

Our study followed standard procedures for studying agency with single modalities [1, 12, 88, 95]. Since smell has never been studied in terms of agency, we based our work on these prior studies to explore if previous found effects on agency modulation occur with smell, unlike other emotion-triggering mechanisms (visual, audio or touch). Yet, we appreciate the complexity of smell compared with other senses for triggering emotions and therefore a key next step, for future work, is exploring SoA with a direct comparison between emotions triggered by smell and emotions triggered by other sensory modalities.

Additionally, since the sense of smell can have a big effect on the overall user experiences (e.g., pleasantness, unpleasantness), we will also explore how scents might affect other aspects of user experience (UX). For example, does a scent increase the feeling of agency but reduce user satisfaction? This possible cross-effects of smell between agency and UX could further inform potential design implications for future olfactory interfaces.

\section{CONCLUSIONS}

In this paper we investigated for the first time the effect of smell on a user's Sense of Agency (SoA). Evidence that agency is modulated by emotions was previously revealed, however to the best of our knowledge, affective modulation has never been explored for agency via the sense of smell. Our results show that smell not only modifies emotions and induces changes in physiological responses, but also affects the feeling of being in control, demonstrated through the use of the IB paradigm. We found that the SoA increased when participants were exposed to a pleasant scent compared to neutral and unpleasant scents. Our findings contribute to the growing research around olfactory interface design and strengthen the importance of the sense of agency concept, giving insights to designers that may be useful when designing for olfactory interfaces that support a sense of being in control.

\section{ACKNOWLEDGMENTS}

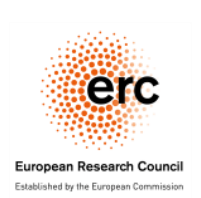

This work was supported by the Mexican National Council of Science and Technology (CONACyT), the European Research Council ERC (grant number 638605 and 737576) and partly supported by the ERC Advanced Grant (number 787413) and the Royal Academy of Engineering through their Chairs in Emerging Technology Program. 


\section{REFERENCES}

[1] Aarts Henk, Bijleveld Erik, Custers Ruud, Dogge Myrthel, Deelder Merel, Schutter Dennis, and Van Haren Neeltje Em, Positive priming and intentional binding: Eye-blink rate predicts reward information effects on the sense of agency. Social Neuroscience, 2012. 7(1): p. 105-112 DOI: https://doi.org/10.1080/17470919.2011.590602

[2] Aggleton John P and Mishkin Mortimer, The amygdala: sensory gateway to the emotions, in Biological foundations of emotion. 1986, Elsevier. p. 281-299 DOI: https://doi.org/10.1016/B978-0-12-558703-7.50018-8

[3] Atsumi Toshiko and Tonosaki Keiichi, Smelling lavender and rosemary increases free radical scavenging activity and decreases cortisol level in saliva. Psychiatry Research, 2007. 150(1): p. 89-96 DOI: https://doi.org/10.1016/j.psychres.2005.12.012

[4] Babcock Linda and Loewenstein George, Explaining bargaining impasse: The role of self-serving biases. Journal of Economic perspectives, 1997. 11(1): p. 109-126 DOI: http://dx.doi.org/10.1037/h0076486

[5] Bandura Albert, Selective moral disengagement in the exercise of moral agency. Journal of moral education, 2002. 31(2): p. 101-119 DOI: https://doi.org/10.1080/0305724022014322

[6] Bandura Albert, Social cognitive theory: An agentic perspective. Annual review of psychology, 2001. 52(1): p. 1-26 DOI: https://doi.org/10.1146/annurev.psych.52.1.1

[7] Barfield Woodrow and Danas Eric, Comments on the use of olfactory displays for virtual environments. Presence: Teleoperators \& Virtual Environments, 1996. 5(1): p. 109-121 DOI: https://doi.org/10.1162/pres.1996.5.1.109

[8] Barkat S, Poncelet J, Landis Basile Nicolas, Rouby C, and Bensafi M, Improved smell pleasantness after odor-taste associative learning in humans. Neuroscience letters, 2008. 434(1): p. 108-112 DOI: https://doi.org/10.1016/j.neulet.2008.01.037

[9] Baron Robert A and Kalsher Michael J, Effects of a pleasant ambient fragrance on simulated driving performance: the sweet smell of... safety? Environment and Behavior, 1998. 30(4): p. $535-552$ DOI: https://doi.org/10.1177/001391659803000407

[10] Barrett And Holland. Available from: https://www.hollandandbarrett.com/

[11] Bergstrom-Lehtovirta Joanna, Coyle David, Knibbe Jarrod, and Hornbæk Kasper. I Really did That: Sense of Agency with Touchpad, Keyboard, and Onskin Interaction. in Proceedings of the 2018 CHI Conference on Human Factors in Computing Systems. $2018 . \quad$ DOI: https://doi.org/10.1145/3173574.3173952

[12] Borhani Khatereh, Beck Brianna, and Haggard Patrick, Choosing, doing, and controlling: implicit sense of agency over somatosensory events. Psychological $\begin{array}{lllll}\text { science, } 2017 . & \mathbf{2 8}(7): & \text { p. } & 882-893 & \text { DOI: }\end{array}$ https://doi.org/10.1177/0956797617697693

[13] Bradley Gifford W, Self-serving biases in the attribution process: A reexamination of the fact or fiction question. Journal of personality and social psychology, 1978. 36(1): p. 56 DOI: http://dx.doi.org/10.1037/00223514.36.1.56

[14] Bradley Margaret M and Lang Peter J, Measuring emotion: the self-assessment manikin and the semantic differential. Journal of behavior therapy and experimental psychiatry, 1994. 25(1): p. 49-59 DOI: https://doi.org/10.1016/0005-7916(94)90063-9

[15] Brewster Stephen, Mcgookin David, and Miller Christopher. Olfoto: designing a smell-based interaction. in Proceedings of the SIGCHI conference on Human Factors in computing systems. 2006. ACM DOI https://doi.org/10.1145/1124772.1124869

[16] Brianza Giada, Tajadura-Jiménez Ana, Maggioni Emanuela, Pittera Dario, Bianchi-Berthouze Nadia, and Obrist Marianna. As light as your scent: effects of smell and sound on body image perception. in IFIP Conference on HumanComputer Interaction. 2019. Springer DOI: https://doi.org/10.1007/978-3030-29390-1 10

[17] Buehner Marc J, Contiguity and covariation in human causal inference. Learning \& Behavior, 2005. 33(2): p. 230-238 DOI: https://doi.org/10.3758/BF03196065

[18] Cameron E Leslie, Pregnancy and olfaction: a review. Frontiers in psychology, 2014. 5: p. 67 DOI: https://doi.org/10.3389/fpsyg.2014.00067

[19] Chrea C, Valentin D, Sulmont-Rossé C, Mai H Ly, Nguyen D Hoang, and Abdi $\mathrm{H}$, Culture and odor categorization: agreement between cultures depends upon the odors. Food Quality and Preference, 2004. 15(7-8): p. 669-679 DOI https://doi.org/10.1016/j.foodqual.2003.10.005

[20] Christensen Jf, Yoshie M, Di Costa S, and Haggard P, Emotional valence, sense of agency and responsibility: A study using intentional binding. Consciousness and cognition, 2016. 43: p. 1-10 DOI https://doi.org/10.1016/j.concog.2016.02.016

[21] Cornelio Martinez Patricia Ivette, De Pirro Silvana, Vi Chi Thanh, and Subramanian Sriram. Agency in mid-air interfaces. in Proceedings of the 2017 CHI Conference on Human Factors in Computing Systems. 2017. ACM DOI: https://doi.org/10.1145/3025453.3025457
[22] Cornelio Martinez Patricia Ivette, Maggioni Emanuela, Hornbæk Kasper, Obrist Marianna, and Subramanian Sriram. Beyond the Libet clock: modality variants for agency measurements. in Proceedings of the ACM CHI Conference on Human Factors in Computing Systems (CHI 2018). 2017. DOI: https://doi.org/10.1145/3173574.3174115

[23] Covarrubias Mario, Bordegoni Monica, Rosini Mauro, Guanziroli Eleonora, Cugini Umberto, and Molteni Franco. VR system for rehabilitation based on hand gestural and olfactory interaction. in Proceedings of the 21st ACM Symposium on Virtual Reality Software and Technology. 2015. ACM DOI: http://dx.doi.org/10.1145/2821592.2821619

[24] Coyle David, Moore James, Kristensson Per Ola, Fletcher Paul, and Blackwell Alan. I did that! Measuring users' experience of agency in their own actions. in Proceedings of the SIGCHI Conference on Human Factors in Computing Systems. 2012. ACM DOI: https://doi.org/10.1145/2207676.2208350

[25] De Groot Jasper Hb, Semin Gün R, and Smeets Monique Am, I can see, hear, and smell your fear: Comparing olfactory and audiovisual media in fear communication. Journal of Experimental Psychology: General, 2014. 143(2): p. 825 DOI: http://dx.doi.org/10.1037/a0033731

[26] Delplanque Sylvain, Coppin Géraldine, and Sander David, Odor and emotion, in Springer handbook of odor. 2017, Springer. p. 101-102 DOI: https://doi.org/10.1007/978-3-319-26932-0 40

[27] Dinh Huong Q, Walker Neff, Hodges Larry F, Song Chang, and Kobayashi Akira. Evaluating the importance of multi-sensory input on memory and the sense of presence in virtual environments. in Virtual Reality, 1999. $\begin{array}{llll}\text { Proceedings. } & \text { IEEE. } & \text { 1999. }\end{array}$ https://doi.org/10.1109/VR.1999.756955

[28] Dmitrenko Dmitrijs, Maggioni Emanuela, Brianza Giada, Holthausen Brittany E, Walker Bruce N, and Obrist Marianna. Caroma therapy: pleasant scents promote safer driving, better mood, and improved well-being in angry drivers. in Proceedings of the 2020 CHI Conference on Human Factors in Computing Systems. 2020. DOI: http://dx.doi.org/10.1145/3313831.3376176

[29] Dmitrenko Dmitrijs, Maggioni Emanuela, and Obrist Marianna. OSpace: towards a systematic exploration of olfactory interaction spaces. in Proceedings of the 2017 ACM International Conference on Interactive Surfaces and Spaces. 2017. ACM DOI: https://doi.org/10.1145/3132272.3134121

[30] Dmitrenko Dmitrijs, Maggioni Emanuela, Vi Chi Thanh, and Obrist Marianna. What did I sniff? Mapping scents onto driving-related messages. in AutomotiveUI'17 Proceedings of the 9th International Conference on Automotive User Interfaces and Interactive Vehicular Applications. 2017. ACM DOI: https://doi.org/10.1145/3122986.3122998

[31] Dong Shan and Jacob Tim Jc, Combined non-adaptive light and smell stimuli lowered blood pressure, reduced heart rate and reduced negative affect. Physiology \& behavior, 2016. 156: p. 94-105 DOI https://doi.org/10.1016/j.physbeh.2016.01.013

[32] Doty Richard L and Cameron E Leslie, Sex differences and reproductive hormone influences on human odor perception. Physiology \& behavior, 2009. 97(2): p. 213-228 DOI: https://doi.org/10.1016/j.physbeh.2009.02.032

[33] Eagleman David M and Holcombe Alex O, Causality and the perception of time. Trends in cognitive sciences, 2002. 6(8): p. 323-325 DOI: https://doi.org/10.1016/S1364-6613(02)01945-9

[34] Ebert Jeffrey P and Wegner Daniel M, Time warp: Authorship shapes the perceived timing of actions and events. Consciousness and cognition, 2010. 19(1): p. 481-489 DOI: https://doi.org/10.1016/j.concog.2009.10.002

[35] Ehrlichman Howard and Bastone Linda, Olfaction and emotion, in Science of olfaction. 1992, Springer. p. 410-438 DOI: https://doi.org/10.1007/978-14612-2836-3 15

[36] Faul Franz, Erdfelder Edgar, Lang Albert-Georg, and Buchner Axel, $G^{*}$ Power 3: A flexible statistical power analysis program for the social, behavioral, and biomedical sciences. Behavior research methods, 2007. 39(2): p. 175-191 DOI: https://doi.org/10.3758/BF03193146

[37] Ferdenzi Camille, Roberts S Craig, Schirmer Annett, Delplanque Sylvain, Cekic Sezen, Porcherot Christelle, Cayeux Isabelle, Sander David, and Grandjean Didier, Variability of affective responses to odors: culture, gender, and olfactory knowledge. Chemical senses, 2013. 38(2): p. 175-186 DOI https://doi.org/10.1093/chemse/bjs083

[38] Ghinea Gheorghita and Ademoye Oluwakemi, The sweet smell of success: Enhancing multimedia applications with olfaction. ACM Transactions on Multimedia Computing, Communications, and Applications (TOMM), 2012 8(1): p. 2 DOI: https://doi.org/10.1145/2663204.2663269

[39] Ghinea Gheorghita and Ademoye Oluwakemi A, Olfaction-enhanced multimedia: perspectives and challenges. Multimedia Tools and Applications, 2011. 55(3): p. 601-626 DOI: https://doi.org/10.1007/s11042-010-0581-4

[40] Greenberg Jeff, Pyszczynski Tom, Burling John, and Tibbs Karyn, Depression, self-focused attention, and the self-serving attributional bias. Personality and Individual Differences, 1992. 13(9): p. 959-965 DOI: https://doi.org/10.1016/0191-8869(92)90129-D

[41] Haggard Patrick, Sense of agency in the human brain. Nature Reviews Neuroscience, 2017. 18(4): p. 196 DOI: https://doi.org/10.1038/nrn.2017.14 
[42] Haggard Patrick, Clark Sam, and Kalogeras Jeri, Voluntary action and conscious awareness. Nature neuroscience, 2002. 5(4): p. 382 DOI: https://doi.org/10.1038/nn827

[43] Haggard Patrick and Tsakiris Manos, The experience of agency: Feelings, judgments, and responsibility. Current Directions in Psychological Science 2009. 18(4): p. 242-246 DOI: https://doi.org/10.1111/j.14678721.2009.01644.x

[44] Harley Daniel, Verni Alexander, Willis Mackenzie, Ng Ashley, Bozzo Lucas, and Mazalek Ali. Sensory vr: Smelling, touching, and eating virtual reality. in Proceedings of the Twelfth International Conference on Tangible, Embedded, and Embodied Interaction. $2018 . \quad$ DOI: https://doi.org/10.1145/3173225.3173241

[45] Harrison Chris, Tan Desney, and Morris Dan. Skinput: appropriating the body as an input surface. in Proceedings of the SIGCHI conference on human factors in computing systems. 2010. ACM DOI: https://doi.org/10.1145/1753326.1753394

[46] Herz Rachel S, Influences of odors on mood and affective cognition. Olfaction, taste, and cognition, 2002. 160: p. 177,

[47] Herz Rachel S and Cupchik Gerald C, The emotional distinctiveness of odorevoked memories. Chemical Senses, 1995. 20(5): p. 517-528 DOI: https://doi.org/10.1093/chemse/20.5.517

[48] Hongratanaworakit Tapanee, Physiological effects in aromatherapy. Songklanakarin J Sci Technol, 2004. 26(1): p. 117-125,

[49] Iscen Ozgun Eylul, Gromala Diane, and Mobini Maryam. Body image and body schema: Interaction design for and through embodied cognition. in International Conference on Human-Computer Interaction. 2014. Springer DOI: https://doi.org/10.1007/978-3-319-07230-2_53

[50] Johansen Katia, Perfumed textiles. Textile Society of America Symposium Proceedings, 2008,

[51] Kaye Joseph Nathaniel, Symbolic olfactory display. 2001, Massachusetts Institute of Technology, http://hdl.handle.net/1721.1/16788

[52] Kaye Joseph" Jofish", Making Scents: aromatic output for HCI. interactions, 2004. 11(1): p. 48-61 DOI: https://doi.org/10.1145/962342.964333

[53] Keller Andreas, Attention and olfactory consciousness. Frontiers in Psychology, 2011. 2: p. 380 DOI: https://doi.org/10.3389/fpsyg.2011.00380

[54] Kolich Michael, In-vehicle smell notification system. 2013, Google Patents,

[55] Komori Teruhisa, Fujiwara Ryoichi, Tanida Masahiro, and Nomura Junichi, Potential antidepressant effects of lemon odor in rats. European Neuropsychopharmacology, 1995. 5(4): p. 477-480 DOI: https://doi.org/10.1016/0924-977X(95)80007-O

[56] Lakens Daniël, Calculating and reporting effect sizes to facilitate cumulative science: a practical primer for t-tests and ANOVAs. Frontiers in psychology, 2013. 4: p. 863 DOI: https://doi.org/10.3389/fpsyg.2013.00863

[57] Lang Peter J, International affective picture system (IAPS): Affective ratings of pictures and instruction manual. Technical report, 2005 , http://csea.phhp.ufl.edu/media.html

[58] Libet Benjamin, Do we have free will? Journal of consciousness studies, 1999. 6(8-9): p. 47-57,

[59] Libet Benjamin, Gleason Curtis A, Wright Elwood W, and Pearl Dennis K, Time of conscious intention to act in relation to onset of cerebral activity (readiness-potential) the unconscious initiation of a freely voluntary act. Brain, 1983. 106(3): p. 623-642 DOI: https://doi.org/10.1093/brain/106.3.623

[60] Limerick Hannah, Coyle David, and Moore James W, The experience of agency in human-computer interactions: a review. Frontiers in human neuroscience, 2014. 8: p. 643 DOI: https://doi.org/10.3389/fnhum.2014.00643

[61] Limerick Hannah, Moore James W, and Coyle David. Empirical evidence for a diminished sense of agency in speech interfaces. in Proceedings of the 33rd Annual ACM Conference on Human Factors in Computing Systems. 2015. ACM DOI: https://doi.org/10.1145/2702123.2702379

[62] Maggioni Emanuela, Cobden Robert, Dmitrenko Dmitrijs , and Marianna Obrist. Smell-O-Message: Integration of Olfactory Notifications into a Messaging Application to Improve Users' Performance. in 2018 International Conference on Multimodal Interaction (ICMI '18). 2018. Boulder, CO, USA: ACM, New York, NY, USA DOI: https://doi.org/10.1145/3242969.3242975

[63] Maggioni Emanuela, Cobden Robert, Dmitrenko Dmitrijs, Hornbæk Kasper, and Obrist Marianna, SMELL SPACE: Mapping out the Olfactory Design Space for Novel Interactions. ACM Transactions on Computer-Human Interaction (TOCHI), 2020. 27(5): $\quad$ p. $1-26 \quad$ DOI: https://doi.org/10.1145/3402449

[64] Matsukura Haruka, Yoneda Tatsuhiro, and Ishida Hiroshi, Smelling screen: development and evaluation of an olfactory display system for presenting a virtual odor source. IEEE transactions on visualization and computer graphics, 2013. 19(4): p. 606-615 DOI: https://doi.org/10.1109/TVCG.2013.40

[65] Mezulis Amy H, Abramson Lyn Y, Hyde Janet S, and Hankin Benjamin L, Is there a universal positivity bias in attributions? A meta-analytic review of individual, developmental, and cultural differences in the self-serving attributional bias. Psychological bulletin, 2004. 130(5): p. 711 DOI: http://dx.doi.org/10.1037/0033-2909.130.5.711
[66] Mochizuki Arito, Amada Takashi, Sawa Sayuri, Takeda Tadayuki, Motoyashiki Shogo, Kohyama Kazuhiro, Imura Masataka, and Chihara Kunihiro. Fragra: a visual-olfactory VR game. in ACM SIGGRAPH 2004 Sketches. 2004. ACM DOI: https://doi.org/10.1145/1186223.1186377

[67] Moore James W and Fletcher Paul C, Sense of agency in health and disease: a review of cue integration approaches. Consciousness and cognition, 2012. 21(1): p. 59-68 DOI: https://doi.org/10.1016/j.concog.2011.08.010

[68] Moore James W and Haggard Patrick, Intentional binding and higher order agency experience. Consciousness and cognition, 2010. 19(1): p. 490-491 DOI https://doi.org/10.1016/j.concog.2009.11.007

[69] Motomura Naoyasu, Sakurai Akihiro, and Yotsuya Yukiko, Reduction of mental stress with lavender odorant. Perceptual and motor skills, 2001. 93(3): p. 713-718 DOI: https://doi.org/10.2466/pms.2001.93.3.713

[70] Nakamoto Takamichi, Otaguro Shigeki, Kinoshita Masashi, Nagahama Masahiko, Ohinishi Keita, and Ishida Taro, Cooking up an interactive olfactory game display. IEEE Computer Graphics and Applications, 2008. 28(1) DOI: https://doi.org/10.1109/MCG.2008.3

[71] Narumi Takuji, Nishizaka Shinya, Kajinami Takashi, Tanikawa Tomohiro, and Hirose Michitaka. Augmented reality flavors: gustatory display based on edible marker and cross-modal interaction. in Proceedings of the SIGCHI conference on human factors in computing systems. 2011. ACM DOI: https://oi.org/10.1145/1978942.1978957

[72] Nordin S, Brämerson A, Murphy C, and Bende M, A Scandinavian adaptation of the Multi-Clinic Smell and Taste Questionnaire: evaluation of questions about olfaction. Acta oto-laryngologica, 2003. 123(4): p. 536-542 DOI https://doi.org/10.1080/00016480310001411

[73] Obrist Marianna, Gatti Elia, Maggioni Emanuela, Vi Chi Thanh, and Velasco Carlos, Multisensory experiences in HCI. IEEE MultiMedia, 2017. 24(2): p. 913 DOI: https://doi.org/10.1109/MMUL.2017.33

[74] Obrist Marianna, Ranasinghe Nimesha, and Spence Charles, Special issue: Multisensory human-computer interaction. 2017 DOI: https://doi.org/10.1016/j.ijhcs.2017.06.002

[75] Obrist Marianna, Tuch Alexandre N, and Hornbaek Kasper. Opportunities for odor: experiences with smell and implications for technology. in Proceeding of the SIGCHI Conference on Human Factors in Computing Systems. 2014. ACM DOI: http://dx.doi.org/10.1145/2556288.2557008

[76] Plush Folly. Available from: https://plushfolly.com/

[77] Porter Jess, Craven Brent, Khan Rehan M, Chang Shao-Ju, Kang Irene, Judkewitz Benjamin, Volpe Jason, Settles Gary, and Sobel Noam, Mechanism of scent-tracking in humans. Nature neuroscience, 2007. 10(1): p. 27 DOI: http://dx.doi.org/10.1038/nn1819

[78] Ranasinghe Nimesha, Jain Pravar, Thi Ngoc Tram Nguyen, Koh Koon Chuan Raymond, Tolley David, Karwita Shienny, Lien-Ya Lin, Liangkun Yan, Shamaiah Kala, and Eason Wai Tung Chow. Season traveller: Multisensory narration for enhancing the virtual reality experience. in Proceedings of the 2018 CHI Conference on Human Factors in Computing Systems. 2018. DOI: https://doi.org/10.1145/3173574.3174151

[79] Ranasinghe Nimesha, Karunanayaka Kasun, Cheok Adrian David, Fernando Owen Noel Newton, Nii Hideaki, and Gopalakrishnakone Ponnampalam. Digital taste and smell communication. in Proceedings of the 6th international conference on body area networks. 2011. ICST (Institute for Computer Sciences, Social-Informatics and Telecommunications Engineering) DOI https://doi.org/10.4108/icst.bodynets.2011.247067

[80] Rinaldi Luca, Maggioni Emanuela, Olivero Nadia, Maravita Angelo, and Girelli Luisa, Smelling the space around us: Odor pleasantness shifts visuospatial attention in humans. Emotion, 2018. 18(7): p. 971 DOI: $\mathrm{http} / / /$ dx.doi.org/10.1037/emo0000335

[81] Sauter Disa A, Eisner Frank, Calder Andrew J, and Scott Sophie K, Perceptual cues in nonverbal vocal expressions of emotion. Quarterly Journal of Experimental Psychology, 2010. 63(11): p. 2251-2272 DOI: https://doi.org/10.1080/17470211003721642

[82] Sbrollini Marilyn C, Olfactory delights. Journal of Chemical Education, 1987 64(9): p. 799 DOI: https://doi.org/10.1021/ed064p799

[83] Shepherd Gordon M., The Human Sense of Smell: Are We Better Than We Think? PLOS Biology, 2004. 2(5): p. e146 DOI: https://doi.org/10.1371/journal.pbio.0020146

[84] Shneiderman Ben, Designing the user interface: strategies for effective humancomputer interaction. 2010: Pearson Education India,

[85] Smith Barry C, The chemical senses, in The Oxford handbook of philosophy of perception. 2015 https://doi.org/10.1093/oxfordhb/9780199600472.013.045

[86] Soudry Y, Lemogne C, Malinvaud D, Consoli S-M, and Bonfils P, Olfactory system and emotion: common substrates. European annals of otorhinolaryngology, head and neck diseases, 2011. 128(1): p. 18-23 DOI: https://doi.org/10.1016/j.anorl.2010.09.007

[87] Tajadura-Jiménez Ana, Basia Maria, Deroy Ophelia, Fairhurst Merle, Marquardt Nicolai, and Bianchi-Berthouze Nadia. As light as your footsteps. altering walking sounds to change perceived body weight, emotional state and 
gait in Proceedings of the $33 \mathrm{rd}$ annual ACM conference on human factors in computing systems. 2015. DOI: https://doi.org/10.1145/2702123.2702374

[88] Takahata Keisuke, Takahashi Hidehiko, Maeda Takaki, Umeda Satoshi, Suhara Tetsuya, Mimura Masaru, and Kato Motoichiro, It's not my fault: postdictive modulation of intentional binding by monetary gains and losses. $\begin{array}{llllll}\text { PloS one, 2012. 7(12): p. } & \text { e53421 } & \text { DOI }\end{array}$ https://doi.org/10.1371/journal.pone.005342

[89] Tham Wendy Wp, Stevenson Richard J, and Miller Laurie A, The functional role of the medio dorsal thalamic nucleus in olfaction. Brain research reviews, $2009 . \quad 62(1)$ $109-126$

DOI: https://doi.org/10.1016/j.brainresrev.2009.09.007

[90] Vernet-Maury Evelyne, Alaoui-IsmaïLi Ouafae, Dittmar Andre, Delhomme Georges, and Chanel Jacques, Basic emotions induced by odorants: a new approach based on autonomic pattern results. Journal of the autonomic nervous system, 1999. 75(2): p. 176-183 DOI: https://doi.org/10.1016/S01651838(98)00168-4

[91] Warrenburg Stephen, Effects of fragrance on emotions: moods and physiology. Chemical Senses, 2005. 30(suppl 1): p. i248-i249 DOI https://doi.org/10.1093/chemse/bjh208

[92] Weber Sandra T and Heuberger Eva, The impact of natural odors on affective states in humans. Chemical senses, 2008. 33(5): p. 441-447 DOI: https://doi.org/10.1093/chemse/bjn011
[93] Yoshida Mariko, Kato Chie, Kawasumi Mikiko, Yamasaki Hatsuo, Yamamoto Shin, Nakano Tomoaki, and Yamada Muneo. Study on Stimulation Effects for Driver Based on Fragrance Presentation. in MVA. 2011. http://www.mvaorg.jp/Proceedings/2011CD/papers/09-26.pdf

[94] Yoshie Michiko and Haggard Patrick, Effects of emotional valence on sense of agency require a predictive model. Scientific Reports, 2017. 7(1): p. 8733 DOI: https://doi.org/10.1038/s41598-017-08803-3

[95] Yoshie Michiko and Haggard Patrick, Negative emotional outcomes attenuate sense of agency over voluntary actions. Current Biology, 2013. 23(20): p 2028-2032 DOI: https://doi.org/10.1016/j.cub.2013.08.034

[96] Zald David $\mathrm{H}$ and Pardo Jose V, Emotion, olfaction, and the human amygdala: amygdala activation during aversive olfactory stimulation. Proceedings of the National Academy of Sciences, 1997. 94(8): p. 4119-4124 DOI https://doi.org/10.1073/pnas.94.8.4119

[97] Zarzo Manuel, Psychologic dimensions in the perception of everyday odors: pleasantness and edibility. Journal of Sensory Studies, 2008. 23(3): p. 354-376 DOI: https://doi.org/10.1111/j.1745-459X.2008.00160.x

[98] Zhang Emma Yann and Cheok Adrian David. A networked device for reproducing multisensory kissing. in Proceedings of the 2016 workshop on Multimodal Virtual and Augmented Reality. 2016. ACM DOI http://dx.doi.org/10.1145/3001959.3001962 